\title{
Efecto de Disciplina Preventiva en Prácticas Parentales Rela- cionadas a Maltrato de Menores
}

\author{
Disciplina Preventiva to Reduce Parental Practices Related to Child Abuse in \\ Puerto Rico
}

\author{
Camille Vélez-Álamo ${ }^{{ }^{*}}$, Aysha Concepción-Lizardi ${ }^{2}$, \& Rubén Vélez-García ${ }^{3}$ \\ 1 Universidad Albizu, San Juan, Puerto Rico. (D) https://orcid.org/0000-0001-5148-2992 \\ 2 Universidad Albizu, San Juan, Puerto Rico. (D) https://orcid.org/0000-0003-3424-9384 \\ 3 Universidad Albizu, San Juan, Puerto Rico. (D) https://orcid.org/0000-0003-2687-7452 \\ * Correspondencia: cv0225354@sju.albizu.edu.
}

Recibido: 13 julio 2021 | Aceptado: 13 agosto 2021 | Publicado: 19 diciembre 2021

\section{WWW.REVISTACARIBENADEPSICOLOGIA.COM}

\section{Citar como:}

Vélez-Álamo, C., Concepción-Lizardi, A., \& Vélez-García, R. (2021). Efecto de Disciplina Preventiva en prácticas parentales relacionadas a maltrato de menores. Revista Caribeña de Psicología, 5, e5705.

https://doi.org/10.37226/rcp.v5i1.5705

\section{RESUMEN}

Prácticas de crianza inadecuadas relacionadas a la coerción, retiro del amor y control, han sido asociadas en la literatura como un factor de riesgo al maltrato infantil. Mientras, prácticas de crianza adecuadas relacionadas con la expresión de afectos, firmeza y consistencia han sido identificadas como factores protectores. El objetivo de este estudio fue evaluar el efecto del programa de formación parental Disciplina Preventiva, en fortalecer prácticas de crianza (adecuadas) que protegen contra el maltrato infantil y desalentar prácticas de crianza relacionadas con maltrato (inadecuadas). Se realizó un análisis de datos secundarios con una muestra de 301 participantes. Los resultados arrojaron que los participantes reportaron cambios significativos en la puntuación total pre vs. post de las prácticas de crianza y de las prácticas inadecuadas. No se observaron cambios significativos en las prácticas adecuadas. El estatus laboral predijo una proporción estadísticamente significativa del cambio entre las prácticas de crianza reportadas por los participantes del programa. Los hallazgos de esta investigación alientan la implantación de programas parentales en poblaciones vulnerables al maltrato infantil, que, presenten estresores adicionales tales como su estatus laboral. Se sugiere continuar fortaleciendo el programa y continuar la investigación acerca del mismo.

Palabras Claves: prácticas de crianza, programa formación parental, riesgo a maltrato

\begin{abstract}
Inadequate parenting practices related to coercion, love withdrawal, and control, have been associated to child abuse risk factors. Meanwhile, adequate parental practices related to loving expressions, firmness, and consistency have been identified as protective factors. The purpose of this study was to evaluate the effect of the Disciplina Preventiva parental formation program to promote adequate parental practices and to discourage inadequate parental practices related to child abuse risk. A secondary data analysis was held, with a sample of 301 participants. The results showed a significant change in the total score of the pre vs. post of parental practices and in the
\end{abstract}


inadequate parental practices. Meanwhile no significant change in the pre vs post adequate parental practices were observed. Work status predicted a statistically significant proportion of the change between parenting practices reported by program participants. Findings suggest the importance of the implementation of parental programs among child abuse risk populations, particularly those populations experimenting stressors such as work status. Additional investigation is encouraged to improve the program so that it can be implemented among diverse groups in Puerto Rico.

Keywords: child abuse risk, parenting practice, parenting program

\section{INTRODUCCIÓN}

La familia es el primer grupo social encargado de la socialización primaria en la vida de niños y niñas (Jorge \& González, 2017). Representa el agente más significativo, pues a través de la familia, se desarrollan habilidades sociales y emocionales y capacidades esenciales que facilitan su integración al mundo (Cuervo, 2010; Méndez et al., 2013). Darling y Steinberg (1993) añaden que, la familia es el entorno en el cual, se constituyen prácticas de crianza que se convierten en punto de referencia para los menores de edad, permitiendo el desarrollo de sus competencias emocionales, el manejo de estrategias de afrontamiento y el logro de estilos de socialización.

Las prácticas de crianza se definen como los conocimientos y actitudes que asumen padres y madres con respecto a los ambientes físicos y sociales y las oportunidades de aprendizaje de sus hijos en el hogar. Las prácticas de crianza suponen la implantación de una serie de normas que siguen las personas frente al comportamiento de menores a su cargo, siendo portadoras de significaciones sociales (Jorge \& González, 2017).

Existe evidencia científica que valida que ciertas prácticas de crianza no solo tienen efectos positivos en los primeros años de vida de los niños y niñas, sino que sus efectos positivos en el nivel social también se ven reflejados durante la etapa de la adolescencia (Darling \& Steinberg, 1993). Algunas de estas prácticas de crianza hacen referencia a la empatía, brindar apoyo, una adecuada resolución de conflictos, una adecuada comunicación, y el control de conducta a través de límites claros. Estas prácticas de crianza son congruentes con la descripción del estilo de crianza democrático elaboradas por Velarde-Arcos y Ramírez-Flores (2017). Este estilo democrático propone que los padres puedan estar en control de la disciplina y la crianza y a su vez, ser exigentes en la implantación de normas. Estas personas son atentas, afectuosas, y sensibles a las peticiones de atención de sus hijos e hijas y son receptivas a sus necesidades.

Herrman y otros investigadores (2005) han reportado que promover prácticas de crianza positivas es una estrategia útil para mejorar el bienestar y el desarrollo psicosocial de niños y niñas, y para prevenir diversas dificultades psicosociales y de salud mental. Estas prácticas pueden aplicarse desde la etapa de lactancia (0 a 3 años) e infancia (3 a 11 años) y se ha comprobado que reducen el riesgo al maltrato infantil (Departamento de la Familia, 2014).

\section{Maltrato Infantil y Prácticas de Crianza}

Contrario al estilo de crianza democrático, la literatura reconoce otros dos estilos de crianza relacionados al maltrato infantil (Gracia, 2002). Autores como Velarde-Arcos y Ramírez-Flores (2017) describen, por un lado, el estilo autoritario, el cual se caracteriza por la manifestación de un alto grado de control y exigencia, bajos niveles de receptividad, comunicación y afecto y la imposición de normas rígidas y alta exigencia de obediencia. Por otra parte, el segundo estilo de crianza asociado al maltrato infantil es el estilo de crianza permisivo. Velarde-Arcos y Ramírez-Flores (2017), describen que estos padres tienden a mostrar bajos niveles de control, pobre receptividad y expresión del afecto con sus hijos e hijas, e intervienen mínimamente en sus actividades, y rechazan el poder $\mathrm{y}$ el control sobre éstos.

La literatura, destaca que el desempleo se relaciona con algunas dimensiones de prácticas de crianza inadecuadas (falta de empatía y castigo físico). Además, la literatura señala que las dificultades económicas pueden contribuir a un mayor estrés en el ámbito familiar y esto a su vez puede desencadenar en prácticas inadecuadas de crianza (Sánchez-Cesáreo et al., 2018; Velarde-Arcos \& Ramírez-Flores, 2017). 
De acuerdo con el Plan Nacional para la Prevención del Maltrato Infantil Puerto Rico 2014-2024, el desconocimiento o la falta de destrezas de crianza y de disciplina, así como el desconocimiento sobre las etapas del desarrollo infantil, los estresores ambientales y laborales pueden inducir a personas adultas a incurrir en maltrato físico, psicológico o emocional hacia los menores a su cargo (Departamento de la Familia, 2014).

El maltrato infantil y el maltrato familiar representan un serio problema de salud pública (Organización Panamericana de la Salud [OPS], 2002). Datos del U.S. Department of Health \& Human Services (2021) arrojaron que en el año 2019 la tasa de maltrato de menores a nivel de toda la nación americana, incluyendo a Puerto Rico fue de 8.9 por cada 1,000 menores. En el caso de Puerto Rico, esta agencia reportó que la tasa de maltrato infantil para este mismo año fue de 8.3 , lo cual representa un total de 4,738 casos fundamentados de maltrato infantil. Los tipos de maltrato más prevalentes fueron: negligencia $(66.4 \%)$, maltrato psicológico $(47.8 \%)$ y maltrato físico $(23.7 \%)$. Estas cifras colocan a Puerto Rico en la posición número 26 en las tasas de maltrato infantil entre todos los estados de la nación americana.

\section{Programas de Formación Parental}

Los programas de formación o capacitación en prácticas de crianza tienen un efecto amortiguador o protector contra el maltrato infantil precisamente por los beneficios que conlleva para la niñez (Departamento de la Familia, 2014). Tienen como objetivo, precisamente, mejorar el desarrollo positivo de los niños y adolescentes brindándoles a los padres o cuidadores principales conocimientos sobre el desarrollo del niño y enseñándoles habilidades para una crianza eficaz (Morales et al. 2016; Morales \& Vázquez, 2014).

En el contexto internacional se han llevado a cabo variadas investigaciones que evalúan la efectividad de los programas de formación parental para promover destrezas de crianza en personas a cargo de menores. Por ejemplo, Vives-Montero y sus colegas (2017) evaluaron los resultados de un programa de entrenamiento de padres y madres para promover prácticas de crianza aplicado en un contexto socio-comunitario en Granada, España. En el estudio participaron 39 personas adultas a cargo de menores entre los cuatro y ocho años. El grupo de tratamiento asistió a siete sesiones de dos horas cada una aproximadamente semanalmente. El grupo de investigadores arrojaron que el grupo de tratamiento mostró mejoría significativa en la aplicación de estrategias de disciplina. Consiguió adoptar técnicas de disciplina más efectivas y prácticas de crianza más apropiadas, relativas a la consistencia y la reducción de la aplicación de estrategias punitivas y coercitivas. También se detectaron cambios significativos en las variables relacionadas al uso de incentivos, reglas claras, elogios, y una clara expresión de las expectativas por parte de padres y madres. En el grupo control no se apreciaron cambios significativos.

En México, Morales y colegas (2016) evaluaron el efecto del cambio de conducta de los padres y madres y la conducta infantil a partir de su participación en un programa de prácticas de crianza positivas. Un total de 128 personas encargadas de niños y niñas entre las edades de dos a 12 años participaron de ocho sesiones donde se adiestraba a los padres sobre los principios básicos de la conducta, tales como el elogio (refuerzo positivo), la corrección (castigo positivo), ignorar conducta inadecuada (extinción de la conducta mantenida por refuerzo positivo o negativo) o el establecimiento de reglas. Los resultados de la intervención reflejaron un aumento en las prácticas de crianza positivas, observándose que las conductas que los padres utilizaron con mayor frecuencia fueron: el elogio, la interacción social, el establecimiento de reglas, destrezas de comunicación y solución de problemas. Así como también, reflejaron menor uso de castigo físico y mayor uso de la técnica de ignorar.

En Puerto Rico por su parte, Sánchez-Cesáreo y demás colegas (2018), llevaron a cabo un estudio en Puerto Rico en el cual se examinó la efectividad de la intervención adaptada a la población puertorriqueña conocida como Fundamentos de Crianza. Esta intervención tenía el propósito de fortalecer las prácticas de crianzas y reducir el nivel de riesgo del maltrato de menores. Además, pretendían examinar los factores sociodemográficos y contextuales que se relacionan con el riesgo al maltrato y negligencia luego de la intervención. Para completar el estudio, se llevó a cabo un análisis de datos secundario con una muestra de 198 participantes que finalizaron el programa de intervención Fundamentos de Crianza durante los años 2015-2016. 
Los resultados sugieren que la intervención está asociada con un incremento en las estrategias de crianza positivas (empatía, afecto, destrezas de comunicación) entre los participantes de todos los grupos. También tuvo un efecto favorable en la reducción de prácticas relacionadas a la falta de empatía sobre las necesidades del niño y el uso de castigo físico. Según este grupo de autores, estas prácticas se relacionan con riesgo de abuso infantil. Y a su vez, encontraron que algunas dimensiones de prácticas de crianza relacionadas con riesgo al maltrato (falta de empatía, castigo físico, poder) se relaciona con el estatus laboral, particularmente el desempleo.

Por otro lado, Miranda-Alvelo (2014) evaluó los efectos de la intervención para el desarrollo de prácticas parentales implantada por Familias CAPACES conocida como Disciplina Preventiva. La investigadora condujo una investigación con una muestra de comunidad en Puerto Rico ascendente a 20 madres que participaron de este currículo de formación parental. Las participantes eran madres mayormente desempleadas y que vivían bajo niveles de pobreza. La investigadora limitó la muestra del estudio a participantes que tuvieran a cargo menores entre los seis y los 12 años.

Para evaluar el cambio en las prácticas de crianza, Miranda-Alvelo utilizó el Inventario de Prácticas de Crianza diseñado por Bauermeister y sus colegas (Bauermeister et al., 2005). En su estudio esta investigadora identificó cambios significativos en las prácticas de crianza en las participantes luego de culminada la intervención. Pero además encontró, que al medir la frecuencia de las conductas mal adaptativas de los hijos e hijas de las participantes luego de la intervención, estas conductas habían reducido significativamente.

En resumen, las intervenciones o programas enfocados en promover prácticas de crianza han demostrado ser una estrategia efectiva para fortalecer factores protectores vinculados a la crianza positiva, reducir los problemas de conducta y reducir prácticas de crianza asociadas a maltrato de menores.

\section{Marco Teórico}

El Modelo Ecológico permite analizar los factores que influyen en el comportamiento o que aumentan el riesgo de cometer o padecer actos violentos, en particular el maltrato infantil. Este modelo es propuesto por el gobierno de Puerto Rico para abordar y prevenir el maltrato de menores (Departamento de la Familia, 2014). La visión sistémica que ofrece el modelo ecológico sirve para explicar cómo la violencia resulta de la interacción de un conjunto de factores en cuatro niveles: (1) el nivel individual, (2) el nivel interpersonal o familiar, (3) el nivel comunitario y (4) el nivel social (Organización Panamericana de la Salud [OPS], 2002).

De acuerdo con el Modelo Ecológico, para atender el problema del maltrato de menores de manera integral y holística, es necesario identificar, analizar y comprender los factores de riesgo y de protección a la luz de los distintos niveles que inciden en la violencia que se ejerce sobre la niñez y la adolescencia (Departamento de la Familia, 2014; Organización Panamericana de la Salud [OPS], 2002). Por una parte, los factores de riesgo son aquellas características que posee la persona, su familia o su entorno comunitario o social que pueden propiciar la práctica de conductas negativas, antisociales y de riesgo a la vida propia o a la de otros. Mientras, los factores de protección tienen el efecto de proteger a la niñez de la violencia pues son los recursos internos y externos que posee la persona que le ayudan a superar, resistir o aminorar riesgos. Estos factores protectores fomentan el bienestar de la familia y disminuyen la probabilidad de que ocurra el maltrato.

El Modelo Ecológico, según propuesto por el Departamento de la Familia, nos ayuda a identificar las causas y los factores que aumentan o disminuyen los riesgos de la violencia, en particular del maltrato infantil. Pero además nos ayuda a entender la forma que interactúan unos niveles con otros, y con ello, se pueden identificar las estrategias y acciones específicas para combatir el maltrato infantil según los diversos niveles. El Departamento de la Familia propone que, la intervención a nivel relacional con padres, madres y personas encargadas de menores, mediante el desarrollo de prácticas parentales asociadas a un estilo de crianza democrático, es una forma de prevenir el maltrato infantil (Departamento de la Familia, 2014). Esto se logra con intervenciones dirigidas a personas adultas a cargo de menores que faciliten el fortalecimiento de los vínculos familiares y el manejo efectivo de la conducta de los niños y niñas bajo su tutela. 


\section{Justificación}

Estilos parentales relacionados a prácticas de crianza caracterizados por la coerción, falta de afecto, negligencia, permisividad y autoritarismo, están relacionadas con algunas tipologías de maltrato infantil. El maltrato infantil tiene serias repercusiones y consecuencias negativas en la vida de niños y niñas. Las consecuencias van desde baja autoestima, bajo rendimiento académico, hasta graves efectos cognitivos como problemas de atención y de aprendizaje (Alarcón, 2010).

El maltrato infantil es un problema muy complejo que no se puede explicar solamente por la presencia o ausencia de un único factor, sino que se trata de una interacción entre múltiples factores de riesgo (Cimpoes, 2014). El Modelo Ecológico propone que los factores de riesgo, en ausencia de los factores de protección, promueven la incidencia de maltrato de menores (Departamento de la Familia, 2014).

Los programas de formación o capacitación para padres son intervenciones a corto plazo que promueven en padres, madres y personas a cargo de menores, estrategias de crianza como un factor de protección contra el maltrato infantil. Según el Modelo Ecológico propuesto por el Departamento de la Familia, estas intervenciones ocurren en el nivel relacional (Departamento de la Familia, 2014). Estas intervenciones ayudan a padres, madres y otras personas encargadas de menores a desarrollar estrategias para la crianza y la disciplina. Las prácticas de crianza positivas pueden ser un factor protector contra el maltrato infantil pero no necesariamente surgen de forma natural, sino que con frecuencia es necesario adquirirlas mediante algún tipo de entrenamiento. Estos programas de formación parental proponen prácticas de crianza relacionadas a estilos de crianza democráticos como el descrito por Velarde-Arcos y Ramírez-Flores (2017).

Bauermeister y sus colegas (2005), sugieren que personas profesionales deben prestar atención clínica a la presencia de altos niveles de prácticas de crianza inadecuadas y a bajos niveles de prácticas adecuadas cuando trabajan con figuras parentales a cargo de la crianza de menores. Las prácticas adecuadas están relacionadas con una dimensión de aceptación-sensibilidad. Esta dimensión incluye estrategias de aprobación (por ejemplo, alabar, dar por bueno, alentar), aconsejar (por ejemplo: razonar, explicar), motivar (por ejemplo: con el uso de recompensas, pérdida de privilegios), sincronización (por ejemplo, prestar atención, ser sensible y responder a las necesidades de la persona menor de edad) y afecto (por ejemplo, calidez y expresión de afecto en la relación). Por otra parte, las prácticas inadecuadas están relacionadas con una dimensión de la crianza en la que predomina la inconsistencia, el retiro del amor y el control a base de la coerción (como por ejemplo el castigo corporal, gritos e insultos).

El propósito de este estudio fue evaluar el efecto del programa de formación parental Disciplina Preventiva en fortalecer prácticas de crianza (adecuadas) que protegen contra el maltrato infantil y reducir con ello prácticas de crianza relacionadas con maltrato (inadecuadas). Este programa ha sido implantado desde 2001 por la entidad sin fines de lucro de base comunitaria Familias CAPACES Inc. Este es un programa dirigido a fortalecer prácticas adecuadas de crianza y reducir con ello, el riesgo de incurrir en prácticas de crianza inadecuadas relacionadas con riesgo a maltrato de menores. El Programa Disciplina Preventiva desarrollado para población puertorriqueña, es un programa fundamentado en las mejores prácticas y evidencia científica. Está adaptado a la cultura y valores de la población, es implantado por profesionales adiestrados, está basado en la teoría y sus resultados se evidencian empíricamente.

\section{Objetivos}

Los objetivos específicos de este estudio se presentan a continuación:

1. Describir las características sociodemográficas, tales como: edad de los padres, madres, tutores o encargados, escolaridad, estatus laboral y la cantidad de hijos en el hogar.

2. Determinar si existen diferencias significativas entre la puntuación total de las prácticas de crianza (adecuadas e inadecuadas) según reportadas por los participantes del programa de formación parental, antes y después de finalizar el programa.

3. Determinar si existen diferencias significativas específicamente entre las prácticas de crianzas adecuadas, así como las prácticas de crianza inadecuadas según reportadas por los partici- 
pantes del programa de formación parental antes y después de finalizar el programa.

4. Evaluar la relación entre las prácticas de crianzas reportadas por los participantes del programa de formación parental y el estatus laboral de los padres, madres, tutores o encargados.

\section{Hipótesis}

- $\mathrm{H}_{2}$ : Existen diferencias estadísticamente significativas al comparar la puntuación total de las prácticas de crianza (adecuadas e inadecuadas) reportadas por los participantes del programa de formación parental antes y después del programa.

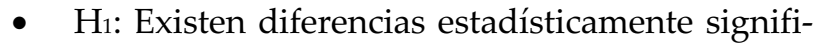
cativas al comparar las prácticas de crianza reportadas por los participantes del programa de formación parental que están a cargo de menores después de finalizar el programa.

- Hza: Las prácticas de crianza adecuadas reportadas.

- $\mathrm{H}_{2 b}$ : Las prácticas de crianza inadecuadas reportadas.

- $\mathrm{H}_{3}$ : El estatus laboral predecirá una proporción estadísticamente significativa del cambio entre las prácticas de crianza reportadas por los participantes del programa de formación parental.

\section{MÉTODO}

\section{Diseño de Investigación}

En el presente estudio se utilizó un diseño de Series de Tiempo Interrumpida. Este diseño se considera uno de los tipos de diseños cuasi experimentales más robustos y se utiliza cuando los diseños experimentales, particularmente la aleatorización, no pueden ser utilizados (Hudson et al., 2019) o cuando las condiciones de campo no posibilitan el uso de grupo control. Este diseño es similar al diseño pretest postest debido al uso de medidas de la variable dependiente antes y después del tratamiento. No obstante, difiere del diseño pretest-postest ya que incluye varias instancias de medidas pre-post (Price et al., 2017). También se utilizó un diseño ex-post-facto para indagar cuáles de las variables independientes contribuyen significativamente a explicar la variabilidad de las diferencias de las puntuaciones totales de prácticas de crianza (Hernández et al., 2014).

\section{Participantes}

Los datos obtenidos para esta investigación fueron obtenidos de una base de datos secundaria de la entidad sin fines de lucro Familias CAPACES Inc. Las personas que completaron los datos para el estudio fueron padres, madres, que participaron del programa de formación parental Disciplina Preventiva cuya característica común era estar a cargo de menores. La muestra de este estudio fue de aproximadamente 301 participantes mayores de 21 años y cuyos hijos o hijas eran menores de 18 años.

\section{Instrumentos}

Hoja de Solicitud de Servicios. Documento creado por Familias CAPACES Inc. que recopila datos socio demográficos, información sobre trabajo, estudios y descripción del núcleo familiar.

Inventario de Prácticas de Crianza (IPC). El IPC es un instrumento de 37 ítems que fue desarrollado por Bauermeister y colegas (2005) para evaluar diferentes aspectos de la crianza y de la disciplina de los niños. El IPC brinda una puntuación total que evalúa la calidad total de las prácticas de crianza y contiene varios reactivos que miden la frecuencia con que los padres, madres o personas encargadas de menores utilizan prácticas de crianza adecuadas e inadecuadas. El IPC es evaluado por la madre, padre o tutor de acuerdo con la siguiente escala: 0 (nunca o casi nunca), 1 (algunas veces), 2 (frecuentemente) y 3 (muy frecuentemente).

Desde el punto de vista clínico, el equipo de investigación de Bauermeister recomienda dar atención especial a si los ítems: 4, 5, 6, 10, 12, 14, 15, 17, 20, 23, 26, $29,30,33$ y 35 fueron evaluados como frecuentemente o muy frecuentemente. Estos ítems componen una dimensión de prácticas de crianza en donde abunda la inconsistencia, el retiro del amor y el control de la conducta a base de la coerción (castigo corporal, gritos, insultos). Estas dimensiones del inventario son congruentes con las conductas relacionadas al maltrato físico, psicológico, emocional y negligencia contra un menor según la Ley para la Seguridad, Bienestar y Protección de Menores (2011). Los demás ítems componen una dimensión de prácticas de crianza denominada aceptación-sensibilidad. Esta dimensión 
incluye estrategias de aprobación (alabar, dar por bueno, alentar), aconsejar (razonar, explicar), motivación (uso de recompensas, pérdida de privilegios), sincronización (prestar atención ser sensible y responsivo) y expresión de los afectos (calor humano) (Barkley et al., 1998). Por lo tanto, si estos ítems son evaluados como nunca, casi nunca y algunas veces, deben recibir atención especial del clínico al interpretar el IPC.

\section{Variables}

Las variables dependientes del estudio fueron las prácticas de crianza reportadas por las personas participantes del programa de formación parental. Como variables independientes se estudiaron: (1) el estatus laboral y (2) la intervención del currículo de formación parental Disciplina Preventiva.

\section{Procedimientos Generales}

Para realizar este estudio, se solicitó la autorización la Junta Directiva de Familias CAPACES Inc., para utilizar un conjunto de la base de datos. La misma solo contenía información de aquellos participantes que consintieron que su información fuera utilizada para investigaciones y estuvo protegida por contraseñas de seguridad. Los datos utilizados en este estudio no incluyeron identificadores personales, cumpliendo así con las guías de protección de confidencialidad de los participantes. El estudio fue aprobado por el Comité para la Ética en la Investigación de la Universidad Carlos Albizu Recinto de San Juan (Protocolo Número: Spring 20-22).

\section{Análisis de Datos}

Se llevaron a cabo análisis univariados para examinar los patrones de respuesta de cada variable independiente bajo estudio. Luego del análisis descriptivo se realizaron análisis bivariados para determinar la relación entre la variable dependiente y las variables independientes. Como requisito de las pruebas de hipótesis, se indagó y evidenció que las puntuaciones de las prácticas de crianza pre y post no cumplían con el supuesto de normalidad ( $p=.001)$. Por esto, se descartó la prueba T student para grupos apareados y se utilizó la prueba no paramétrica de los rangos con signo de Wilcoxon.

La prueba de los rangos con signo de Wilcoxon fue utilizada con el propósito de conocer si la puntuación total de las prácticas de crianza reportadas por los participantes del programa de formación parental difiere antes y después de finalizar el programa. Igualmente, se utilizó para evaluar los efectos del programa de formación parental para promover cambio en las destrezas de crianza de las personas encargadas de menores en dos aspectos. Por un lado, se evaluó el efecto de la intervención en promover un cambio en las puntuaciones totales de las prácticas adecuadas e inadecuadas. También se evaluó el efecto de la intervención en aumentar las prácticas parentales adecuadas y en reducir las prácticas parentales inadecuadas.

Finalmente, se llevó a cabo una regresión lineal múltiple para evaluar si el estatus laboral de los padres se relaciona con el cambio en las prácticas de crianza reportadas por los participantes del programa de formación parental. Los datos fueron analizados utilizando el Paquete Estadístico para las Ciencias Sociales (SPSS, por sus siglas en inglés versión 27.0).

\section{RESULTADOS}

La mayoría de los participantes fueron mujeres, estaban desempleados (65.4\%) y contaban con un nivel académico igual o mayor a escuela superior (Tabla 1). El promedio de edad de los participantes fue de 34.5 años $(D E=11.41)$ y el promedio de hijos en el hogar fue de 1.4 hijos $(D E=1.24)$.

\section{Tabla 1}

\begin{tabular}{llc}
\multicolumn{3}{c}{ Características Sociodemográficas de los Participantes. } \\
\hline Variables & $n$ & $\%$ \\
\hline Género & 255 & $84.7 \%$ \\
Femenino & 43 & $14.3 \%$ \\
Masculino & & \\
$\quad$ Grado Académico & 20 & $8.8 \%$ \\
Menor a escuela superior & 83 & $36.7 \%$ \\
Igual a escuela superior & 123 & $54.4 \%$ \\
$\quad$ Mayor a escuela superior & & \\
Estatus Laboral & 197 & $65.4 \%$ \\
$\quad$ No trabaja & 110 & $34.6 \%$ \\
$\quad$ Si trabaja
\end{tabular}

Los resultados de la prueba de los rangos con signo de Wilcoxon muestran que existe una diferencia estadísticamente significativa al comparar la puntuación total de las prácticas de crianza reportadas por los participantes (inadecuadas y adecuadas) 
antes y después de la intervención de formación parental, $T=3889.50, \mathrm{SET}_{\mathrm{T}}=317.10, \mathrm{z}=3.32, p=.001$. Los resultados también sugieren que existen diferencias estadísticamente significativas entre las prácticas de crianza inadecuadas reportadas antes y después del programa, $T=6035.50, \mathrm{SET}_{\mathrm{T}}=394.89, \mathrm{z}=5.62, p=.001$. En relación con las prácticas adecuadas, se observó que no existen diferencias estadísticamente significativas según reportadas antes y después de la participación en el programa, $T=4423.00, \mathrm{SE} \mathrm{T}=386.33, \mathrm{z}=$ $1.89, p=.06$.

Así mismo, los resultados de los análisis de regresión muestran que el modelo propuesto fue significativo $F(1,88)=4.617, p=.03$, explicando un $5.0 \%$ de la varianza de las diferencias del cambio de las puntuaciones totales de las prácticas de crianza. En dicho modelo, la variable estatus laboral demostró contribuir significativamente a la explicación de la variabilidad de las diferencias del cambio de las puntuaciones de las prácticas de crianza $(t=-2.149, p=.03)$. El modelo final fue el siguiente:

\section{Dif Post-Pre (Prácticas de Crianza) $=2.49$ (Estatus Laboral) -5.86 . \\ DISCUSIÓN}

El objetivo de este estudio fue evaluar el efecto del programa de formación parental Disciplina Preventiva en las prácticas parentales de crianza en personas adultas a cargo de menores de 18 años. Este programa ha sido implantado y desarrollado por la entidad sin fines de lucro Familias CAPACES Inc. para población puertorriqueña. El programa está dirigido a fortalecer prácticas adecuadas de crianza en personas a cargo de menores y reducir con ello, la probabilidad de incurrir en prácticas de crianza inadecuadas que representan un riesgo de maltrato infantil. También se evaluó el efecto del estatus laboral sobre el cambio en las prácticas reportadas.

Los resultados sugieren que el programa Disciplina Preventiva se asocia con un cambio positivo, tanto en la puntuación total (adecuadas e inadecuadas) de las prácticas de crianza, como en las prácticas inadecuadas de crianza en personas participantes a cargo de menores. Estos hallazgos son consistentes con los de Miranda-Alvelo (2014) quien encontró que el programa Disciplina Preventiva tuvo un efecto positivo en promover prácticas de crianza en una muestra de comunidad compuesta de 20 mujeres a cargo de menores entre los seis y los 12 años de edad.

Es decir, por un lado, las personas encargadas de menores reportaron una mejoría significativa en las prácticas de crianza totales que representan la dimensión de aceptación-sensibilidad (prácticas adecuadas) y en la dimensión en la que predomina la inconsistencia, el retiro del amor y el control a base de la coerción (inadecuadas). Pero, además, al considerar las prácticas parentales inadecuadas asociadas con la inconsistencia, el retiro del amor y el control de la conducta a base de la coerción se encontró que hubo una reducción en estas. Esto quiere decir que padres, madres y personas a cargo de menores luego de la intervención desarrollaron estrategias (prácticas adecuadas) de aprobación (por ejemplo, alabar, dar por bueno, alentar), aconsejar (por ejemplo: razonar, explicar), motivar (por ejemplo: con el uso de recompensas, pérdida de privilegios), sincronización (por ejemplo prestar atención, ser sensible y responder a las necesidades de la persona menor de edad) y afecto (por ejemplo calidez y expresión de los afectos en la relación). Pero estas personas, a su vez redujeron prácticas inadecuadas las cuales están relacionadas con una dimensión de la crianza en la que predomina la inconsistencia, el retiro del amor y el control a base de la coerción (como por ejemplo el castigo corporal, gritos e insultos).

Estos hallazgos nos llevan a retomar el modelo ecológico desarrollado por Urie Bronfenbrenner y adoptado por el Departamento de la Familia para la prevención del maltrato infantil (Departamento de la Familia, 2014). Este propone que, para prevenir el maltrato infantil, hemos de considerar intervenciones en los múltiples niveles en que se relacionan padres, madres, y sus hijos e hijas, el ambiente familiar, la comunidad en que viven y la sociedad. El currículo de formación parental Disciplina Preventiva ejerce su mayor influencia en el nivel familiar-relacional del Modelo Ecológico. Este nivel familiar-relacional hace referencia a las interacciones entre las díadas paterno y materno filial. Pero a su vez, estas diadas paterno y materno filial, ejercen sus influencias sobre otras personas, díadas, y sub-sistemas como la escuela, la comunidad (Departamento de la Familia, 2014). Podemos decir que, si en una diada materno o paterno filial mejora la capacidad de ser sensibles, de demostrarse los afectos, de prestarse atención mutuamente, 
de alentarse, de motivarse y de razonar, ello puede ejercer una influencia positiva sobre otros sub-sistemas y sus componentes tales como pares, vecinos, pareja y amistades en la familia, la escuela o comunidad en que se desenvuelven las personas. Por lo tanto, estas influencias positivas sobre la calidad de los vínculos y las relaciones al interior de la familia, puede incidir y reducir el riesgo de la violencia relacional y comunitaria.

Adquiere relevancia el hallazgo de que personas encargadas de menores hayan reducido las prácticas de crianza inadecuadas al interior de la familia relacionadas a la inconsistencia, el retiro del amor y el control a base de la coerción (como por ejemplo el castigo corporal, gritos e insultos). Estas prácticas han sido relacionadas a lo largo de la literatura al maltrato infantil (Gracia, 2002; Velarde-Arcos \& Ramírez-Flores, 2017). Específicamente son prácticas asociadas a las tipologías de maltrato físico, maltrato psicológico y maltrato por negligencia. Estas tipologías de maltrato han ido en aumento en la última década. Reducir la probabilidad de que un padre, madre o persona encargada de una persona menor de edad incurra en este tipo de maltrato que tiene un impacto sobre el bienestar y la vida de un menor, puede salvaguardar la vida o el bienestar socioemocional de niños y niñas a corto y largo plazo. Además, al considerar en este análisis el Modelo Ecológico, podemos decir que estos resultados desalientan prácticas "culturales" adoptadas como normales tales como el castigo físico. Ciertamente el castigo físico no genera alternativas de conducta en los menores de edad. Todo lo contrario, habitúa a los menores al castigo y desalienta conductas adaptativas futuras (Vives-Montero et al., 2017).

Los resultados de este estudio reflejaron que, aunque reportaron cambios significativos en las prácticas de crianza adecuadas e inadecuadas, padres, madres, y otras personas a cargo de menores no reportaron cambios en las prácticas adecuadas basadas únicamente en prácticas de crianza en las que predomina la aprobación, motivación, sincronización y afecto. Es decir que no se identificó un cambio significativo de las prácticas parentales adecuadas relacionadas únicamente a estilos democráticos en la crianza.

Por otra parte, los resultados arrojaron que el estatus laboral explica una proporción significativa de las prácticas de crianza. Las personas desempleadas a cargo de menores reflejaron un aumento en las prácticas de crianza. Esto implica una mejoría en sus prácticas de crianza. Ello es consistente con los hallazgos de Miranda-Alvelo (2014) quien encontró que el programa del Programa de formación parental Disciplina Preventiva se asocia con un efecto positivo en el desarrollo de destrezas de crianza con una muestra de comunidad compuesta por 20 mujeres, en su mayoría bajo el nivel de pobreza (85\%) y desempleadas (80\%). Estos resultados aportan evidencia científica acerca de los efectos positivos de programas de formación parental en contextos comunitarios y con diversos grupos que representan diversidad socioeconómica.

Este estudio presenta varias limitaciones. La primera de ellas es la identificación de los valores perdidos. De los 301 casos evaluados, aproximadamente un $36 \%$ completó en su totalidad la pre-prueba y post-prueba de la intervención. Esto dificulta la generalización de los datos y evaluar el mantenimiento de las prácticas de crianza de los participantes a largo plazo (Lozano-Rodríguez \& Valero-Aguayo, 2017). Por otra parte, no se limitaron las edades de los menores al considerar la muestra. Es decir, se incluyeron padres, madres y encargados con menores entre uno y 18 años.

Los resultados de este estudio contribuyen a ofrecer recomendaciones dirigidas a promover evaluación adicional del currículo de formación parental Disciplina Preventiva. Sería útil evaluar los efectos de la intervención del currículo de formación parental específicamente con personas a cargo de menores entre las edades de cero a 11 años. La literatura recomienda que es precisamente en estas etapas del desarrollo cuando es más efectivo promover prácticas de crianza positivas como estrategia útil para mejorar el funcionamiento familiar, y para promover el bienestar y el desarrollo psicosocial de niños y niñas (Departamento de la Familia, 2014).

Además, sería recomendable implantar en el futuro diseños experimentales para evaluar los efectos del currículo de formación parental Disciplina Preventiva. Los estudios experimentales permitirían hacer comparaciones entre grupos, controlar otras variables bajo estudio e identificar cómo estas impactan las prácticas de crianza. Entre las variables que señala la literatura que pueden ser controladas se encuentran la edad de los hijos e hijas de las personas participan- 
tes, nivel socioeconómico, educación y estatus marital (Sánchez-Cesáreo et al., 2018). Resultados de los estudios controlados, podrían generar conocimiento adicional sobre la efectividad de este programa de formación parental desarrollado para población puertorriqueña. A su vez, ello podría ofrecer una alternativa de servicios que se adapte a padres, madres y personas encargadas de menores en Puerto Rico beneficiarias de otras organizaciones gubernamentales, comunitarias y profesionales comprometidas con la prevención del abuso infantil en Puerto Rico. De esta forma, el programa podría ser ampliado y replicado en contextos diversos y con poblaciones diversas. Esto supondría además que este currículo pudiera generar una alternativa que promueva el auto sustento para la entidad sin fines de lucro Familias CAPACES Inc.

La literatura científica ha evidenciado que los programas comunitarios de formación parental son beneficiosos en lograr cambios en las prácticas o estilos de crianza, y por ende en disminuir el riesgo maltrato infantil y transformar el funcionamiento familiar (Vives-Montero et al., 2017). El Programa Disciplina Preventiva fue diseñado para ser implantado en el contexto comunitario y en los ambientes naturales de las familias. Las intervenciones en contextos comunitarios son recursos valiosos pues acercan los servicios a las familias y menores vulnerables. Con estas intervenciones comunitarias, se apoya a familias en desarrollar mejores prácticas de crianza que promuevan la calidad de la relación en la díada paterno y materno filial, y con ello desalentar prácticas de crianza relacionadas al maltrato infantil.

Se sugiere que se continúe la difusión de la investigación sobre este programa para evaluar de forma sistemática cómo atiende los factores asociados al riesgo de incurrir en prácticas de crianza adecuadas e inadecuadas. Particularmente se recomienda que se continúe la investigación acerca de la implantación del currículo en comunidades y poblaciones con altos niveles de desempleo que presenten estresores relacionados a ello, tales como falta de accesos a servicios de salud y de salud mental. Y que, a su vez, se desarrollen políticas públicas e iniciativas dirigidas al desarrollo de programas que promuevan la autogestión, el autocuidado y que puedan reducir los niveles de desempleo en personas a cargo de menores de edad. Y con ello, la reducción de los estresores relacionados al desempleo que impactan la diada materno, paterno filial. Además, se recomienda que el currículo pueda adaptarse y flexibilizarse y pueda ser un espacio de respiro para padres, madres y personas encargadas de menores que enfrentan el estresor de la doble jornada: trabajo no remunerado en la casa con hijos e hijas y trabajo remunerado fuera de casa.

\section{CONCLUSIÓN}

La literatura sugiere que, personas a cargo de menores participen en programas de formación parental que promuevan sus prácticas de crianza para reducir el riesgo de maltrato infantil. Al evaluar el efecto del programa de formación parental Disciplina Preventiva para fortalecer prácticas de crianza, este estudio encontró que el programa fue efectivo en promover cambios significativos en la puntuación total pre vs. post de las prácticas de crianza de las personas participantes adultas a cargo de menores. En particular, el estatus laboral predijo una proporción estadísticamente significativa del cambio en las prácticas de crianza reportadas por los participantes del programa. Los hallazgos de este estudio alientan la investigación acerca del currículo Disciplina Preventiva y su implantación con personas a cargo de menores que presentan altos niveles de desempleo, falta de accesos a servicios de salud y de salud mental. Los hallazgos promueven, a su vez, el desarrollo de políticas públicas e iniciativas dirigidas a la creación e implantación de programas que promuevan la autogestión, el autocuidado y que puedan reducir los niveles de desempleo en personas a cargo de menores de edad.

Este estudio es una aportación al campo de la ciencia de la psicología en Puerto Rico. Por un lado, permitió acercar la ciencia al servicio al evaluar científicamente una práctica basada en evidencia desarrollada y diseñada para población puertorriqueña por una entidad sin fines de lucro de base comunitaria. Además, representa una alternativa culturalmente viable para personas adultas a cargo de menores para la prevención del maltrato de menores en Puerto Rico.

Financiamiento: La presente investigación no fue financiada por alguna entidad ni patrocinador.

Conflicto de Intereses: No existen conflictos de intereses de parte de los autores de la investigación. 
Aprobación de la Junta Institucional para la Protección de Seres Humanos en la Investigación: Comité para la Ética en la Investigación de la Universidad Carlos Albizu Recinto de San Juan (Protocolo Número: Spring 20-22).

Consentimiento o Asentimiento Informado: No fue necesario; se realizaron análisis de datos secundarios.

Proceso de Revisión: Este estudio ha sido revisado por pares externos en modalidad de doble ciego.

\section{REFERENCIAS}

Alarcón, L. C., Araujo, A. P., Godoy, A. P., \& Vera, M. E. (2010). Maltrato infantil y sus consecuencias a largo plazo. MedUNAB, 13, 103-115.

Bauermeister, J. J., Matos, M., Reina, G., Salas, C., Martínez, J. V., Cumba, E., \& Barkley, R. (2005). Comparison of the DSM-IV Combined and Inattentive Types of ADHD in a School-Based Sample of Latino/Hispanic Children. Journal of Child Psychology and Psychiatry, 46 (2), 166-179.

Cimpoes, A (2014). Factores de riesgo y prevención del maltrato infantil. [Trabajo Final de Grado, Universitat Jaume]. http://hdl.handle.net/10234/103037

Concepción Lizardi, A., \& Sánchez-Peraza, L.R. (2017). Familias CAPACES: Alternativas ecológicas al manejo del referido en casos de maltrato de menores en Puerto Rico. Revista Puertorriqueña de Psicología, 28(1), 172-188.

Cuervo, A. (2010). Pautas de crianza y desarrollo socioafectivo en la infancia. Revista Diversitas-Perspectivas en Psicología, 6(1), 111- 121.

Darling, N., \& Steinberg, L. (1993). Parenting style as context: An integrative model. Psychological Bulletin, 113(3), 487-496. http://dx.doi.org/10.1037/0033-2909.113.3.487

Departamento de la Familia. (2014). Plan Nacional para la Prevención de Maltrato de Menores en Puerto Rico 2014-2024. http://www.acuden.pr.gov/Programa\%20Child\%20Care/Documents/Ninez\%20Temprana/Plan\%20nacional\%20para\%20la\%20prevenci $\%$ C3\%B3n\%20del\%20maltrato\%20de\%20menores\%20en\%20Puerto\%20Rico\%202014-2024.pdf.

Hernández, R., Fernández, C., \& Baptista, M. P. (2014). Metodología de la Investigación. McGraw-Hill.

Herrman, H., Saxena, S., \& Moodie, R. (2005). Promoting Mental Health: Concepts, Emerging Evidence, Practice. A Report from the World Health Organization, Department of Mental Health and Substance Abuse in collaboration with the Victorian Health Promotion Foundation and The University of Melbourne. https://www.who.int/mental_health/evidence/en/promoting_mhh.pdf

Hudson, J., Fielding, S., \& Ramsay, C. R. (2019). Methodology and reporting characteristics of studies using interrupted time series design in healthcare. BMC Medical Research Methodology, 19(1). https://doi.org/10.1186/s12874-019-0777-x

Jorge, E. \& González, C. (2017). Estilos de crianza parental: una revisión teórica. Informes Psicológicos, 17(2), 39-66. http://dx.doi.org/10.18566/infpsic.v17n2a02

Lozano-Rodríguez, I., \& Valero-Aguayo, L. (2017). Una revisión sistemática de la eficacia de los programas de entrenamiento a padres. Revista de Psicología Clínica con Niños y Adolescentes, 4 (2), 85-101.

Méndez, M., Andrade, P., \& Peñaloza, R. (2013). Prácticas parentales y capacidades y dificultades en preadolescentes. Revista Intercontinental de Psicología y Educación, 15(1), 99-118.

Miranda Alvelo, D. K. (2014). Evaluación de la efectividad del currículo de talleres de formación parental Disciplina Preventiva de la Organización Familias CAPACES Inc. [Disertación doctoral no publicada]. Universidad Carlos Albizu.

Morales-Chainé, S., \& Vázquez, F. (2014). Prácticas de crianza asociadas a la reducción de los problemas de conducta infantil: Una aportación a la salud pública. Acta De Investigación Psicológica, 4 (3), 1700- 1715.

Morales-Chainé, S., Martínez, M. J., Martin, R., \& Nieto, J. (2016). Las prácticas de crianza y la reducción de los problemas de conducta infantil. Behavioral Psychology, 24 (2), 341-357.

Organización Panamericana de la Salud [OPS], Oficina Regional para las Américas de la Organización Mundial de la Salud. (2002). Informe mundial sobre la violencia y la salud: resumen. Washington, D.C.

Price, P. C., Jhangiani R. S., Chiang C. A., Leighton, D. C., \& Cuttler, C. (2017). Research Methods in Psychology. (3 ${ }^{\text {rd }}$ ed.). https://opentext.wsu.edu/carriecuttler/chapter/8-1-one-groupdesigns/

Sánchez-Cesáreo, M., Sánchez-Cardona, I., González-Jiménez, C., \& Bensinger, K. (2018). Factores asociados al abuso de menores: Resulados de una intervención para el fortalecimiento de prácticas de crianza. Revista Puertorriqueña de Psicología, 29(1) 16-35.

U.S. Department of Health \& Human Services, Administration for Children and Families, Administration on Children, Youth and Families, Children's Bureau. (2021). Child Maltreatment 2019. https://www.acf.hhs.gov/cb/research-data-technology/statistics-research/child-maltreatment

Velarde-Arcos, M.P., \& Ramírez-Flores, M.J. (2017). Efectos de las prácticas de crianza en el desempeño cognitivo en niños de edad preescolar. Rev. Chil. Neuropsicol, 12(1), 12-18. 10.5839/rcnp.2017.12.01.04

Vives-Montero, C., Cortés-Pendón, D., López-Rubio, S., \& Ascanio, L. (2017). Efectos de un programa de formación de padres para reducir conductas perturbadoras infantiles. Revista de Psicopatología y Psicología Clínica, 22(3), 219-227. 